\title{
Vegetation Ecology Associated with Some Species of Family Zygophyllaceae in Different Biogeographic Regions of Egyptian Desert
}

\author{
Mohamed Abd El-Aal, Ibrahim A. Mashaly ${ }^{*}$, Magda I. Soliman, Rehab M. Rizk, Mona F. Elmorsy \\ Botany Department, Faculty of Science, Mansoura University, Mansoura, Egypt
}

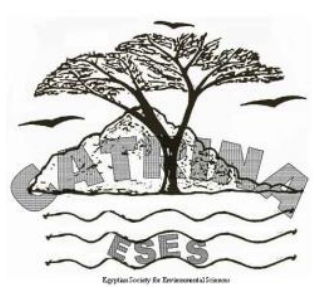

\begin{abstract}
The Zygophyllaceae is a widespread family of common existence in arid and semi-arid habitats of the Old and New Worlds, especially in seasonally dry deserts. In the present study, eight species of Zygophyllaceae namely, Fagonia arabica L., Fagonia bruguieri DC., Fagonia cretica L., Fagonia mollis Delile, Zygophyllum aegyptium Hosny, Zygophyllum coccineum L., Zygophyllum decumbens Delile, and Zygophyllum simplex L. were selected. The floristic features and chorological affinities of the selected species with their associates in relation to soil variables were investigated. The sampling was carried out in 38 sampled stands within two types of deserts in Egypt; the inland Eastern desert and the coastal desert of Mediterranean Sea coast. A total of 133 plant species belonging to 29 families and 107 genera were recorded. Asteraceae, Poaceae, Chenopodiaceae, Brassicaceae, Fabaceae, and Zygophyllaceae were the most species-rich families which represented by approximately $60.15 \%$ of the total number of the investigated species. Preponderance of perennials $(60 \%)$, therophytes $(40 \%)$ and Saharo-Sindian/ Mediterranean taxa (62\%) was remarkable indicating the arid and semi-arid climate of the investigation territory. Based on the importance value, four vegetation groups were identified after the application of TWINSPAN. Group A, was dominated by Zygophyllum aegyptium and represented sand formations habitat; group B, was dominated by Fagonia cretica and represented road-side habitats of the western Mediterranean coastal desert; group C, was dominated by Zygophyllum coccineum and represented Wadi Hagul bed and gravel desert habitats of Suez and group D, was dominated by Pulicaria undulata and represented wadi bed and roadside habitats in Ismailia. Ordination of the vegetation groups along 2 axes was performed using Detrended Correspondence Analysis (DCA), while Canonical Correspondence Analysis (CCA) exhibited that calcium carbonate, organic carbon, pH, electrical conductivity, chlorides, total nitrogen, total soluble phosphorus and cations ( $\mathrm{Na}, \mathrm{K}, \mathrm{Ca} \& \mathrm{Mg}$ ) were the most important soil variables that affecting the abundance of vegetation groups.
\end{abstract}

Keywords: Chorotype, Classification, Desert, Medicinal plants, Ordination, Soil, Zygophyllaceae.

\section{INTRODUCTION}

Over recent decades, the majority of the natural flora of the Egyptian desert was influenced by human activities, for example, development of the deltaic part of wadis, over-grazing, the intensive gathering of plant species for its values (fuel, fiber, therapeutic, and so on.), and establishment of new towns, industrial facilities, streets, highways, infrastructure, water pipelines, and quarries. These activities cause desert degradation and fragmentation and a damaging impact in the plant diversity. This implies a great alteration in the presence, distribution, and extinction of the desert plants (Abd El-Ghani et al., 2017; Abdelaal, 2017).

The phytogeographical regions in Egypt are seven regions as reported by Boulos (2009), these include: 1 . The Nile region including the Delta, Valley and Faiyum, 2. The Oases of the Western Desert including Wadi Natrun, Siwa, Farafra, Bahariya, Kharga, Dakhla, Kurkur, Dungul and Uweinat, 3. The Mediterranean coastal strip extending from Sallum at west to Rafah at east, 4.The Desert including Western and Eastern Deserts, 5. The Red Sea coastal strip, 6. Gebel Elba and the surrounding mountainous region and 7. Sinai Peninsula.

Zygophyllaceae, the caltrop family, is a group of angiosperm plants frequently restricted to tropical and warm climates. It is widespread in Old and New Worlds especially arid and semi-arid zones. Members of this family are mostly shrubby, but some are herbs and rarely some grow as trees. According to the classifica- tion by Sheahan and Chase (1996 \& 2000), Zygophyllaceae is a heterogeneous family represented by approximately 27 genera and 285 plant species. The taxonomy within the family has changed over time, since they display a large amount of variability in structural detail (Abdel Khalik, 2012). At the family level, numerous studies were carried out in order to elucidate the systematic position of most of its members (Sheahan and Cutler, 1993; Sheahan and Chase, 1996; Beier et al., 2003). However, Engler (1896 \& 1931) divided the family into seven subfamilies. Nevertheless, Sheahan and Chase (1996 \& 2000) divided Zygophyllaceae into five subfamilies.

In Egypt, Zygophyllaceae is represented by three subfamilies namely: Peganoideae Engl., Tetradiclidoideae Engl., and Zygophylloideae; which encompass of six genera (Täckholm, 1974; El Hadidi ,1972). However, recently five genera were recorded by Boulos (2000).

In this study, two major genera of Zygophyllaceae were selected: Zygophyllum and Fagonia. Zygophyllum; which considered as the largest genus of Zygophyllaceae with about 80 to 100 species distributed in warm, seasonally dry areas of Asia, Africa, and Australia. It is a genus of succulent plants found as shrubs, subshrubs, and herbs, mostly with bifoliolate leaves. In the flora of Egypt, Zygophyllum is broadly distributed in Desert, Sinai, Oases, Gebel Elba, Red Sea and Mediterranean area (Täckholm, 1974; Boulos, 2000). The abundance of genus Zygophyllum is 
dependent on its tolerance to the different environmental stresses, while its distribution is correlated to structure of soil in its habitats (Mashaly et al., 2015). Many species of Zygophyllum are known as economic and medicinal plants that characterized by antidiabetic, anthelmintic, antihyperglycemic, antihypertensive and insecticidal effects and exhibit anti-inflammatory, antimicrobial and antioxidant activity (Gibbons and Oriowo, 2001; El Ghoul et al., 2012; Elbadry et al., 2015). Regardless of the significance of Zygophyllum in addition to its abundance in the Mediterranean and Middle East areas, there isn't sufficient studies and information about them.

Fagonia is a widespread genus represented by 34 species growing in warm and arid zones in all continents except Australia, with a center of distribution in northern Africa and the Middle East, especially in the Saharo-Sindian and Mediterranean regions. It consists of low shrubs and woody perennials often with spinescent or pointed stipules and opposite, simple or 37-foliolate leaves. Fagonia is often recognized from Zygophyllum by its spinescent stipules, usually trifoliolate leaves (however some Fagonia species vary from one-foliolate to multifoiolate) (Sheahan and Chase, 2000; Beier et al., 2004). In Egypt, with its central position in Saharo-Sindian region, Fagonia includes 18 species recorded by El Hadidi (1966) and Täckholm (1974) but recently 15 species were reported by Boulos (2000) and Abdel Khalik, (2012).

This genus is of great concern to many researchers and pharmacists, since it contains many active const-ituents of medicinal and pharmaceutical significance. It is often used as a popular remedy in folk medicine for the treatment of various ailments. Traditionally, Fagonia is well known as hepatic tonic for many liver diseases and for the treatment of number of different ailments such as anemia, small-pox, diabetes, joint pain, dropsy, jaundice, cough, asthma, against poisoning and for the treatment of various skin lesions. It is claimed to contain potential anticancer agents acting against breast cancer cell (Lam et al., 2012; Pareek et al., 2013; Qureshi et al., 2016; Farheen et al., 2017).

The selected species are Fagonia arabica L., Fagonia bruguieri DC., Fagonia cretica L., Fagonia mollis Delile, Zygophyllum aegyptium Hosny, Zygophyllum coccineum L., Zygophyllum decumbens Delile, and Zygophyllum simplex L.

This study intended to investigate the spatial distribution, habitat conditions, ecological aspects and chorological affinity of the selected Zygophyllaceae species with their associated plant species in some phytogeographic regions of Egyptian desert.

\footnotetext{
MATERIALS AND METHODS

\section{Study area}

Deserts cover about $20 \%$ of the land area of the world. In Egypt, It covers approximately $95 \%$ of the total area of the country. Desert vegetation is almost certainly the most characteristic and important type of the natural vegetation. The Egyptian deserts are among the most arid areas of the world. Hence, desert vege-tation covers
}

immense zones formed mainly of xerophy-tes shrubs and subshrubs (Abd El-Ghani et al 2017). The sampled stands (38) were distributed within coastal desert along the Middle and Western Medi-terranean coast and in the inland Eastern desert (Figure: 1).

The coastal deserts in Egypt extend along the Mediterranean, the Red Sea, and the Gulfs of Suez and Aqaba in Sinai, hence and because of the different distribution, massive variations in the floristic composition and vegetation structure occur. The Mediterranean coast of Egypt expanses between Rafah on the Egyptian-Palestinian border and Sallum on the Egyptian-Libyan border for about $970 \mathrm{~km}$ in three sections: Sinai coast (the eastern section), Deltaic coast (the middle section), and finally Mareotis coast (the western section). In general, this region of Egypt belongs to the dry arid zone and the Mediterranean type climate (Zahran and Willis, 2009). The Middle Mediterranean coast extends for about $220 \mathrm{~km}$ from Port-Said to Abu-Qir. It can be distinguished into four main habitats: sand formations, salt marshes, sandy lands and reed swamps (Mashaly, 2001 \& 2002).

On the other hand, The Western Mediterranean coastal land stretches for about $550 \mathrm{~km}$ from Abu-Qir to Sallum. It represents by far the richest biogeographic region of Egypt; with about 50\% of the total of the Egyptian flora, because of the relatively high rainfall. Most of these species are therophytes. According to Zahran and Willis (2009), it is partitioned into sand dunes, rocky ridges, saline depressions, barley fields and inland plateau habitats.

The Eastern Desert of Egypt occupies about $21 \%$ of the total area of the country. It is distinguished into two main ecological units, the inland Eastern Desert with its wadis, and the Red Sea costal land. The inland Eastern Desert extends for about $223,000 \mathrm{~km}^{2}$ between the Red Sea coastal mountains in the east and the Nile Valley in the west. It is a rocky plateau dissected by a number of wadis. It can be divided into four main geomorphological and ecological regions: 1) Cairo-Suez Desert, 2) Limestone Desert, 3) Sandstone Desert, and 4) Nubian Desert (Zahran and Willis, 2009).

\section{Floristic sampling}

After several field trips during spring 2016, a total of 38 stands $(10 \times 10 \mathrm{~m}$ each $)$ were selected along coastal desert and inland desert. Plant specimens from the different stands were collected and brought to the Herbarium of Botany Department, Faculty of Science, Mansoura University for identification and voucher specimens were preserved. Identification and nomenclature of the investigated plant species were carried out as stated by Boulos (1999-2009). The chorological analysis was according to Zohary (1966 \& 1972) and Feinbrun-Dothan (1978 \& 1986), while the description and classification of life-forms were followed that of Raunkiaer (1934).

In each stand, the importance value (IV, out of 200) was estimated for the different plant species by calculating the sum of the relative values of density and cover (Mueller-Dombois and Ellenberg, 1974). 


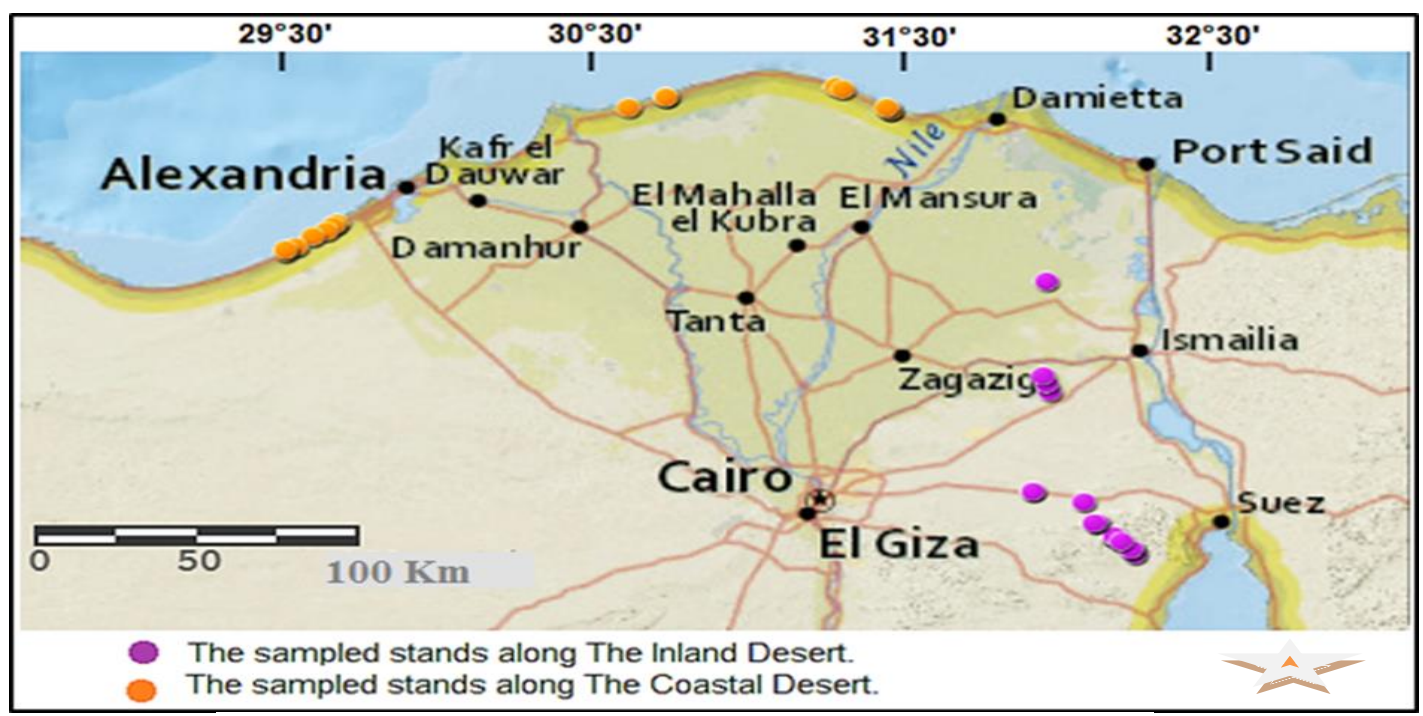

Figure: 1 The location of the sampled stands.

\section{Soil sampling}

Soil samples were collected from each stand at 0-50 $\mathrm{cm}$ depth, allowed to dry in air, well mixed and then sieved to remove any debris and gravel from soil. The chemical variables estimated in this present study were calcium carbonate, organic carbon, $\mathrm{pH}$, electrical conductivity, anions $\left(\mathrm{Cl}^{-}, \mathrm{SO}_{4}^{--}\right.$and $\left.\mathrm{HCO}_{3}{ }^{-}\right)$, total nitrogen, total dissolved phosphorous, and extractable cations $\left(\mathrm{Na}^{+}, \mathrm{K}^{+}, \mathrm{Ca}^{++}\right.$and $\left.\mathrm{Mg}^{++}\right)$. Calcium carbonate was estimated by dissolving in $\mathrm{HCl}$ then using titration against $1 \mathrm{~N} \mathrm{NaOH}$. Oxidizable organic carbon was quantified using Walkley-Black rapid method (1934). Extract $(1: 5 \mathrm{w} / \mathrm{v})$ of each soil sample was prepared for the record of soil reaction $(\mathrm{pH})$ and electrical conductivity (EC) using $\mathrm{pH}$-meter and conductivity meter, respectively. Chloride content was determined by direct titration against silver nitrates using potassium chromates indicator. Sulphates were gravimetrically assessed using barium chloride solution to be participated as barium sulphate (Wilde et al., 1964). Soluble bicarbonates content was appraised by titration method with $0.1 \mathrm{~N} \mathrm{HCl}$ and using methyl orange indicator. Total nitrogen was valued using the air dry samples by the conventional semi-micromodification of Kjeldahl method, while total phosphorus was determined by direct stannous chloride method. The extractable cations were approximated in each soil sample according to AOAC (1990). A Flame Photometer was used to determine sodium and potassium cations, whereas calcium and magnesium were estimated by an Atomic Absorption Spectrometer (Upadhyay and Sharma, 2005).

\section{Data analysis}

Two Way Indicator Species Analysis (TWINSPAN) was operated to categorize the floristic records of 133 species in 38 stands using Community Analysis Package (CAP) program (Henderson and Seaby 2007), while Detrended Correspondence Analysis (DCA) was directed to ordinate the sampled stands along the first 2 axes. Canonical Correspondence Analysis (CCA) was used to determine the relation between the leading characteristic species and the measured soil factors (Ter Braak, 2003).

\section{RESULTS}

\section{Floristic features}

The floristic features of the eight selected plant species with their associates in the present study were displayed in Table 1. In total, 133 plant species belonging to 29 families and 107 genera were recorded in the 38 sampled stands. The largest species-rich families were Asteraceae (23 species), Poaceae (17), Chenopodiaceae (13), Brassicaceae, Fabaceae and Zygophyllaceae families ( 9 for each). The remaining 23 families were represented together by 53 species; in miniature representation $7,6,5,4,3,2$ or one species). Fagonia and Zygophyllum were the largest representative genera in the surveyed study with four species for each. Out of 133 species, 80 species (60\%) were perennials, 49 species $(37 \%)$ were annuals, and 4 species (3\%) were biennials (Fig. 2).

As displayed in Figure 3, the life-form spectrum therophytes $(53$ species $=39.85 \%)$ were the dominant, followed by chamaephytes ( 37 species $=27.82 \%)$, hemicryptophytes $(24$ species $=18.05 \%)$, phanerophytes $(12$ species $=9.02 \%)$, geophytes $(5$ species $=3.76 \%)$, cryptophytes ( 1 species $=0.75 \%$ ), and parasites (one species $=0.75 \%)$.

After excluding the three cultivated and naturalized plant species, the chorotypes of the recorded plant species were presented in Table (2) and Figure (4). Biregional elements achieved the major contribution by $40 \%$ (52 species) of the total number of species, followed by monoregional elements ( 43 species $=33 \%$ ), 
Table (1): Associated species of the surveyed plant species with their families, life span, life-forms and chorotypes.

\begin{tabular}{|c|c|c|c|c|}
\hline Species & Family & Life span & Life form & Chorotype \\
\hline Achillea fragrantissima (Forssk.) Sch.Bip. & Asteraceae & Per & $\mathrm{Ch}$ & SA-SI+IR-TR \\
\hline Achillea santolina $\mathrm{L}$. & Asteraceae & Per & $\mathrm{Ch}$ & SA-SI+IR-TR \\
\hline Alhagi graecorum Boiss. & Fabaceae & Per & $\mathrm{H}$ & PAL \\
\hline Anabasis articulata (Forssk.) Moq. & Chenopodiaceae & Per & $\mathrm{Ch}$ & SA-SI+IR-TR \\
\hline Anchusa humilis (Desf.) I. M. Johnst. & Boraginaceae & Ann & $\mathrm{Th}$ & $\mathrm{ME}+\mathrm{SA}-\mathrm{SI}$ \\
\hline Arthrocnemum macrostachyum (Moric.) K. Koch. & Chenopodiaceae & Per & $\mathrm{Ch}$ & $\mathrm{ME}+\mathrm{SA}-\mathrm{SI}$ \\
\hline Atractylis carduus (Forssk.) C. Chr. & Asteraceae & Per & $\mathrm{H}$ & SA-SI+ME \\
\hline Atriplex halimus $\mathrm{L}$. & Chenopodiaceae & Per & $\mathrm{Ph}$ & $\mathrm{ME}+\mathrm{SA}-\mathrm{SI}$ \\
\hline A. lindleyi Moq. & Chenopodiaceae & Ann & $\mathrm{Th}$ & AUST \\
\hline A. semibaccata $\mathrm{R}$. Br. & Chenopodiaceae & Per & $\mathrm{H}$ & AUST \\
\hline Avena fatua $\mathrm{L}$. & Poaceae & Ann & Th & PAL \\
\hline Bassia indica (Wight) A. J.Scott. & Chenopodiaceae & Ann & $\mathrm{Th}$ & S-Z+IR-TR \\
\hline Beta vulgaris $\mathrm{L}$. & Chenopodiaceae & $\mathrm{Bi}$ & Th & ME+IR-TR+ER-SR \\
\hline Brassica tournefortii Gouan & Brassicaceae & Ann & Th & $\mathrm{ME}+\mathrm{IR}-\mathrm{TR}+\mathrm{SA}-\mathrm{SI}$ \\
\hline Bromus diandrus Roth & Poaceae & Ann & $\mathrm{Th}$ & ME \\
\hline B. rubens $\mathrm{L}$. & Poaceae & Ann & Th & ME+IR-TR \\
\hline Cakile maritima Scop. & Brassicaceae & Ann & Th & ME+ER-SR \\
\hline Calligonum polygonoides L. & Polygonaceae & Per & $\mathrm{Ph}$ & SA-SI+IR-TR \\
\hline Calotropis procera (Aiton) W.T. Aiton & Asclepiadaceae & Per & $\mathrm{Ph}$ & SA-SI+S-Z \\
\hline Carrichtera anпиа (L.) DC. & Brassicaceae & Ann & Th & SA-SI \\
\hline Carthamus tenuis (Boiss. \& Blanche) Bornm. & Asteraceae & Ann & Th & ME \\
\hline Caylusea hexagyna (Forssk.) M. L. Green & Resedaceae & Ann & Th & SA-SI+S-Z \\
\hline Centaurea aegyptiaca $\mathrm{L}$. & Asteraceae & $\mathrm{Bi}$ & Th & SA-SI \\
\hline C. alexandrina Delile & Asteraceae & $\mathrm{Bi}$ & $\mathrm{Th}$ & SA-SI \\
\hline Chenopodium murale L. & Chenopodiaceae & Ann & $\mathrm{Th}$ & COSM \\
\hline Cistanche phelypaea (L.) Cout. & Orobanchaceae & Per & $\mathrm{Pa}$ & SA-SI+ME \\
\hline Citrullus colocynthis (L.) Schrad. & Cucurbitaceae & Per & $\mathrm{Ch}$ & CULT and NAT \\
\hline Cleome amblyocarpa Barratte \& Murb. & Cleomaceae & Ann & Th & SA-SI+IR-TR \\
\hline Convolvulus lanatus Vahl & Convolvulaceae & Per & $\mathrm{Ch}$ & SA-SI \\
\hline Conyza bonariensis (L.) Cronquist & Asteraceae & Ann & $\mathrm{Th}$ & NEO \\
\hline Cornulaca monacantha Delile & Chenopodiaceae & Per & $\mathrm{Ch}$ & SA-SI+IR-TR \\
\hline Crotalaria aegyptiaca Benth. & Fabaceae & Per & $\mathrm{Ch}$ & SA-SI \\
\hline Cutandia memphitica (Spreng.) Benth. & Poaceae & Ann & $\mathrm{Th}$ & $\mathrm{ME}+\mathrm{IR}-\mathrm{TR}+\mathrm{SA}-\mathrm{SI}$ \\
\hline Cynanchum acutum $\mathrm{L}$. & Asclepiadaceae & Per & $\mathrm{H}$ & ME+IR-TR \\
\hline Cynodon dactylon (L.) Pers. & Poaceae & Per & G & PAN \\
\hline Deverra tortuosa (Desf.) DC. & Apiaceae & Per & $\mathrm{Ch}$ & SA-SI \\
\hline Diplotaxis harra (Forssk.) Boiss. & Brassicaceae & Per & $\mathrm{H}$ & $\mathrm{ME}+\mathrm{SA}-\mathrm{SI}$ \\
\hline Echinops spinosus L. & Asteraceae & Per & $\mathrm{H}$ & $\mathrm{ME}+\mathrm{SA}-\mathrm{SI}$ \\
\hline Echium angustifolium Mill. & Boraginaceae & Per & $\mathrm{H}$ & $\mathrm{ME}$ \\
\hline E. sericeum (Vahl) Klotz & Boraginaceae & Per & $\mathrm{H}$ & ME \\
\hline Elymus farctus (Viv.) Runem. ex Melderis & Poaceae & Per & G & ME \\
\hline Emex spinosa (L.) Campd. & Polygonaceae & Ann & Th & $\mathrm{ME}+\mathrm{SA}-\mathrm{SI}$ \\
\hline Erodium glaucophyllum (L.) L'Hér. & Geraniaceae & Per & $\mathrm{H}$ & ME \\
\hline E. laciniatum (Cav.) Willd. & Geraniaceae & Ann & $\mathrm{Th}$ & $\mathrm{ME}$ \\
\hline Eryngium creticum Lam. & Apiaceae & Per & $\mathrm{H}$ & ME+IR-TR \\
\hline Euphorbia peplus L. & Euphorbiaceae & Ann & Th & ME+IR-TR+ER-SR \\
\hline E. terracina $\mathrm{L}$. & Euphorbiaceae & Per & $\mathrm{H}$ & ME \\
\hline Fagonia arabica $\mathrm{L}$. & Zygophyllaceae & Per & $\mathrm{Ch}$ & SA-SI \\
\hline
\end{tabular}


Table 1. Continued.

\begin{tabular}{|c|c|c|c|c|}
\hline Species & Family & Life span & Life form & Chorotype \\
\hline F. bruguieri DC. & Zygophyllaceae & Per & $\mathrm{Ch}$ & SA-SI \\
\hline F. cretica $\mathrm{L}$. & Zygophyllaceae & Per & $\mathrm{Ch}$ & ME \\
\hline F. mollis Delile & Zygophyllaceae & Per & $\mathrm{Ch}$ & SA- SI \\
\hline Farsetia aegyptia Turra subsp. aegyptia & Brassicaceae & Per & $\mathrm{Ch}$ & SA-SI+S-Z \\
\hline Gymnocarpos decandrus Forssk. & Caryophyllaceae & Per & $\mathrm{Ch}$ & SA-SI \\
\hline Gypsophila capillaris (Forssk.) C. Chr. & Caryophyllaceae & Per & $\mathrm{H}$ & SA-SI+IR-TR \\
\hline Halocnemum strobilaceum (Pall.) M. Bieb. & Chenopodiaceae & Per & $\mathrm{Ch}$ & ME+IR-TR+SA-SI \\
\hline Haloxylon salicornicum (Moq.) Bunge ex Boiss. & Chenopodiaceae & Per & $\mathrm{Ch}$ & SA-SI \\
\hline Heliotropium arbainense Fresen. & Boraginaceae & Per & $\mathrm{Ch}$ & SA-SI \\
\hline H. digynum (Forssk.) Asch. Ex C. Chr. & Boraginaceae & Per & $\mathrm{Ch}$ & SA-SI \\
\hline Herniaria hemistemon $\mathrm{J}$. Gay & Caryophyllaceae & Per & $\mathrm{H}$ & $\mathrm{ME}+\mathrm{SA}-\mathrm{SI}$ \\
\hline Hordeum marinum Huds. & Poaceae & Ann & Th & $\mathrm{ME}+\mathrm{IR}-\mathrm{TR}+\mathrm{ER}-\mathrm{SR}$ \\
\hline Hyoscyamus muticus $\mathrm{L}$. & Solanaceae & Per & $\mathrm{Ch}$ & SA-SI+IR-TR \\
\hline Ifloga spicata (Forssk.) Sch. Bip. subsp. spicata & Asteraceae & Ann & Th & $\mathrm{ME}+\mathrm{SA}-\mathrm{SI}$ \\
\hline Imperata cylindrica (L.) Raeusch. & Poaceae & Per & $\mathrm{H}$ & $\mathrm{ME}+\mathrm{PAL}$ \\
\hline Iphiona mucronata (Forssk.)Asch. & Asteraceae & Per & $\mathrm{Ch}$ & SA-SI \\
\hline Juncus acutus L. & Juncaceae & Per & $\mathrm{He}$ & $\mathrm{ME}+\mathrm{IR}-\mathrm{TR}+\mathrm{ER}-\mathrm{SR}$ \\
\hline J. rigidus Desf. & Juncaceae & Per & $\mathrm{H}$ & ME+SA-SI+IR-TR \\
\hline Kickxia aegyptiaca (L.) Nabelek. & Schrophulariaceae & Per & $\mathrm{Ch}$ & $\mathrm{ME}+\mathrm{SA}-\mathrm{SI}$ \\
\hline Lasiurus scindicus Henrard. & Poaceae & Per & $\mathrm{Cr}$ & SA-SI+S-Z \\
\hline Launaea mucronata (Forssk.) Musch1. & Asteraceae & Per & $\mathrm{H}$ & ME+SA-SI \\
\hline L. nudicaulis (L.) Hook.f. & Asteraceae & Per & $\mathrm{H}$ & SA-SI \\
\hline L. spinosa (Forssk.)Sch.Bip. ex Kuntze. & Asteraceae & Per & $\mathrm{Ch}$ & SA-SI \\
\hline Lavandula coronopifolia Poir. & Lamiaceae & Per & $\mathrm{Ch}$ & SA-SI \\
\hline Lepidium draba $\mathrm{L}$. & Brassicaceae & Per & $\mathrm{H}$ & $M E+I R-T R$ \\
\hline Leptadenia pyrotechnica (Forrsk.) Decne. & Asclepiadaceae & Per & $\mathrm{Ph}$ & SA-SI \\
\hline Limonium pruinosum (L.) Chaz. & Plumbaginaceae & Per & $\mathrm{H}$ & SA-SI \\
\hline Lolium multiflorum Lam. & Poaceae & Ann & Th & ME+IR-TR+ER-SR \\
\hline Lotus deserti Täckh. \& Boulos & Fabaceae & Ann & Th & SA-SI \\
\hline L. glaber Mill. & Fabaceae & Per & $\mathrm{H}$ & $\mathrm{ME}+\mathrm{IR}-\mathrm{TR}+\mathrm{ER}-\mathrm{SR}$ \\
\hline L. halophilus Boiss. \& Spruner & Fabaceae & Ann & Th & $\mathrm{ME}+\mathrm{SA}-\mathrm{SI}$ \\
\hline Lycium shawii Roem. \& Schult. & Solanaceae & Per & $\mathrm{Ph}$ & SA-SI+S-Z \\
\hline Malva parviflora $\mathrm{L}$. & Malvaceae & Ann & Th & $\mathrm{ME}+\mathrm{IR}-\mathrm{TR}$ \\
\hline Matthiola longipetala (Vent.)DC. & Brassicaceae & Ann & Th & $M E+I R-T R$ \\
\hline Mesembryanthemum crystallinum $\mathrm{L}$. & Aizoaceae & Ann & Th & ME+ER-SR \\
\hline M. forsskaolii Hochst. ex Boiss. & Aizoaceae & Ann & Th & SA-SI \\
\hline M. nodiflorum $L$. & Aizoaceae & Ann & Th & ME+SA-SI+ER-SR \\
\hline Nicotiana glauca R. C. Graham & Solanaceae & Per & $\mathrm{Ph}$ & CULT and NAT \\
\hline Ochradenus baccatus Delile & Resedaceae & Per & $\mathrm{Ph}$ & SA-SI \\
\hline Ononis serrata Forssk. & Fabaceae & Ann & Th & $\mathrm{ME}+\mathrm{SA}-\mathrm{SI}$ \\
\hline O. vaginalis Vahl & Fabaceae & Per & $\mathrm{Ch}$ & IR-TR+ SA-SI \\
\hline Onopordum alexandrinum Boiss. & Asteraceae & $\mathrm{Bi}$ & Th & IR-TR+ SA-SI \\
\hline Pancratium maritimum $\mathrm{L}$. & Amaryllidaceae & Per & G & ME \\
\hline Panicum turgidum Forssk. & Poaceae & Per & $\mathrm{H}$ & SA-SI \\
\hline Parapholis incurva (L.) C.E. Hubb & Poaceae & Ann & Th & $\mathrm{ME}+\mathrm{IR}-\mathrm{TR}+\mathrm{ER}-\mathrm{SR}$ \\
\hline Phalaris minor Retz. & Poaceae & Ann & Th & ME+IR-TR \\
\hline Phragmites australis (Cav.) Trin.ex Steud. & Poaceae & Per & $\mathrm{H}$ & COSM \\
\hline Picris asplenioides $\mathrm{L}$. & Asteraceae & Ann & Th & $\mathrm{ME}+\mathrm{IR}-\mathrm{TR}$ \\
\hline Plantago lagopus L. & Plantaginaceae & Ann & Th & ME \\
\hline
\end{tabular}


Table 1. Continued.

\begin{tabular}{|c|c|c|c|c|}
\hline Species & Family & life span & life form & chorotype \\
\hline Pluchea dioscoridis (L.) DC. & Asteraceae & Per & $\mathrm{Ph}$ & $\mathrm{SA}-\mathrm{SI}+\mathrm{S}-\mathrm{Z}$ \\
\hline Polygonum equisetiforme Sibthi \& Sm. & Polygonaceae & Per & $\mathrm{G}$ & ME+IR-TR \\
\hline Polypogon monspeliensis (L.) Desf. & Poaceae & Ann & Th & COSM \\
\hline Pulicaria incisa (Lam.) DC. & Asteraceae & Per & $\mathrm{Ch}$ & SA-SI \\
\hline P. undulata (L.) C.A.Mey. & Asteraceae & Per & $\mathrm{Ch}$ & SA-SI+S-Z \\
\hline Reaumuria hirtella Jaub. \& Spach var. hirtella & Tamaricaceae & Per & $\mathrm{Ch}$ & IR-TR+ SA-SI \\
\hline Reichardia tingitana (L.) Roth & Asteraceae & Ann & $\mathrm{Th}$ & $\mathrm{ME}+\mathrm{IR}-\mathrm{TR}+\mathrm{SA}-\mathrm{SI}$ \\
\hline Reseda decursiva Forssk. & Resedaceae & Ann & Th & SA-SI \\
\hline Retama raetam (Forssk.) Webb\& Berthel. & Fabaceae & Per & $\mathrm{Ph}$ & $\mathrm{ME}+\mathrm{IR}-\mathrm{TR}+\mathrm{SA}-\mathrm{SI}$ \\
\hline Ricinus communis $\mathrm{L}$. & Euphorbiaceae & Per & $\mathrm{Ph}$ & CULT and NAT \\
\hline Rumex pictus Forssk. & Polygonaceae & Ann & $\mathrm{Th}$ & ME+SA-SI \\
\hline R. vesicarius L. & Polygonaceae & Ann & Th & $\mathrm{ME}+\mathrm{SA}-\mathrm{SI}+\mathrm{S}-\mathrm{Z}$ \\
\hline Salsola kali $\mathrm{L}$. & Chenopodiaceae & Ann & Th & COSM \\
\hline Salvia deserti Decne. & Lamiaceae & Per & $\mathrm{Ch}$ & SA-SI+IR-TR \\
\hline Schismus barbatus (L.) Thell. & Poaceae & Ann & Th & ME+IR-TR+SA-SI \\
\hline Scrophuloria deserti Delile & Schrophulariaceae & Per & $\mathrm{Ch}$ & SA-SI \\
\hline Senecio glaucus L. & Asteraceae & Ann & Th & $\mathrm{ME}+\mathrm{IR}-\mathrm{TR}+\mathrm{SA}-\mathrm{SI}$ \\
\hline Silene succulenta Forssk. & Caryophyllaceae & Per & $\mathrm{H}$ & ME \\
\hline S. viviani Steud. & Caryophyllaceae & Ann & $\mathrm{Th}$ & $\mathrm{ME}+\mathrm{SA}-\mathrm{SI}$ \\
\hline Sisymbrium irio $\mathrm{L}$. & Brassicaceae & Ann & Th & ME+IR-TR+ER-SR \\
\hline Sonchus oleraceus L. & Asteraceae & Ann & Th & COSM \\
\hline Spergularia salina J. \& C. Presl & Caryophyllaceae & Ann & Th & $\mathrm{ME}+\mathrm{IR}-\mathrm{TR}+\mathrm{ER}-\mathrm{SR}$ \\
\hline Stipagrostis lanata (Forssk.) De Winter & Poaceae & Per & G & SA-SI \\
\hline Suaeda vera Forssk. ex J.F. Gmel. & Chenopodiaceae & Per & $\mathrm{Ch}$ & ME+SA-SI+ER-SR \\
\hline Symphyotrichum squamatum (Spreng.) Nesom & Asteraceae & Per & $\mathrm{Ch}$ & NEO \\
\hline Tamarix nilotica (Ehrenb.) Bunge & Tamaricaceae & Per & $\mathrm{Ph}$ & $\mathrm{SA}-\mathrm{SI}+\mathrm{S}-\mathrm{Z}$ \\
\hline Thymelaea hirsuta (L.) Endl. & Thymelaceae & Per & $\mathrm{Ph}$ & ME \\
\hline Tribulus terrestris L. & Zygophyllaceae & Ann & Th & ER-SR+ME+IR-TR \\
\hline Trichodesma africanum (L.) R.Br. & Boraginaceae & Per & $\mathrm{Ch}$ & SA-SI+S-Z \\
\hline Trigonella stellata Forssk. & Fabaceae & Ann & $\mathrm{Th}$ & SA-SI+IR-TR \\
\hline Urospermum picroides (L.) F.W. Schmidt & Asteraceae & Ann & Th & ME+IR-TR \\
\hline Zilla spinosa (L.) Prantl & Brassicaceae & Per & $\mathrm{Ch}$ & SA-SI \\
\hline Zygophyllum aegyptium Hosny & Zygophyllaceae & Per & $\mathrm{Ch}$ & ME \\
\hline Z. coccineum $\mathrm{L}$. & Zygophyllaceae & Per & $\mathrm{Ch}$ & SA-SI+S-Z \\
\hline Z. decumbens Delile & Zygophyllaceae & Per & $\mathrm{Ch}$ & SA-SI \\
\hline Z. simplex $\mathrm{L}$. & Zygophyllaceae & Ann & $\mathrm{Th}$ & PAL \\
\hline Ononis serrata Forssk. & Fabaceae & Ann & Th & $\mathrm{ME}+\mathrm{SA}-\mathrm{SI}$ \\
\hline O. vaginalis $\mathrm{Vahl}$ & Fabaceae & Per & $\mathrm{Ch}$ & IR-TR+ SA-SI \\
\hline Onopordum alexandrinum Boiss. & Asteraceae & $\mathrm{Bi}$ & Th & IR-TR+ SA-SI \\
\hline Pancratium maritimum $\mathrm{L}$. & Amaryllidaceae & Per & G & ME \\
\hline
\end{tabular}

Ann: annuals; Per: perennials; Bi: biennials; Th: therophyte; Ch: chamaephyte; $\mathrm{H}$ : hemicryptophytes; Ph: phanerophytes; Cr: cryptophytes; P: parasites; ME: Mediterranean; SA-SI: Saharo-Sindian; IR-TR: Irano-Turanian; S-Z: Sudano-Zambezian; ER-SR: Euro-Siberian; PAL: Palaeotropical, COSM: Cosmopolitan; PAN: Pantropical; NEO: Neotropical; AUST: Australian, CULT and NAT: Cultivated and Naturalized 


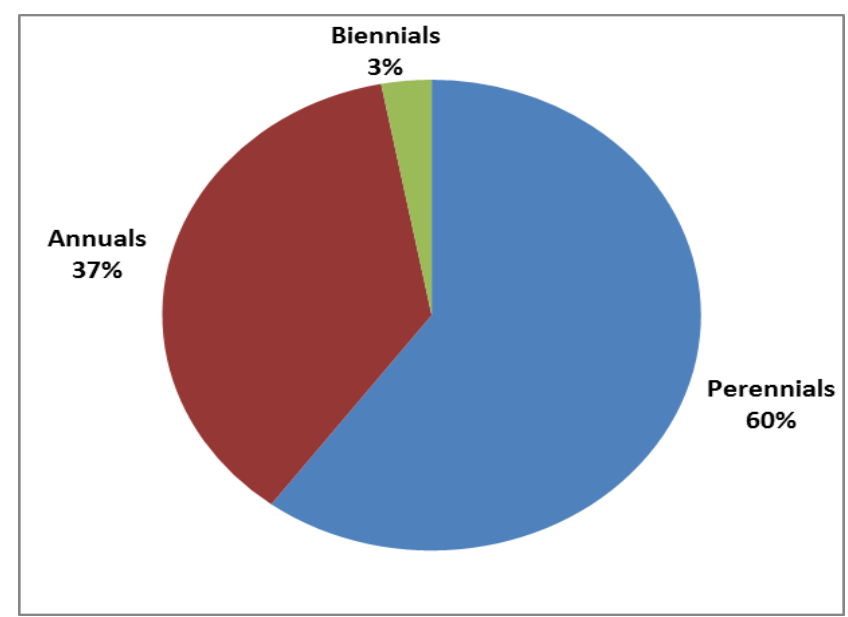

Figure (2): Life-span (\%) of the surveyed plant species in the present study.

Table (2): Chorological analysis (Number of species and percentage) in the present study

\begin{tabular}{lcc}
\hline \hline Chorotype & Number & \% \\
\hline ME & Mono-regional elements & \\
SA-SI & 14 & $10.77 \%$ \\
Sub-total & 29 & $22.31 \%$ \\
& $\mathbf{4 3}$ & $\mathbf{3 3 \%}$ \\
SA-SI+IR-TR & Bi-regional elements & \\
S-Z+IR-TR & 13 & $10 \%$ \\
SA-SI+S-Z & 1 & $0.77 \%$ \\
ME+SA-SI & 10 & $7.69 \%$ \\
ME+IR-TR & 16 & $12.31 \%$ \\
ME+ER-SR & 10 & $7.69 \%$ \\
Sub-Total & 2 & $1.54 \%$ \\
& $\mathbf{5 2}$ & $\mathbf{4 0 \%}$ \\
ME+IR-TR+ER-SR & 10 & $7.69 \%$ \\
ME+IR-TR+SA-SI & 8 & $6.15 \%$ \\
ME+SA-SI+ER-SR & 2 & $1.54 \%$ \\
ME+SA-SI+S-Z & 1 & $0.77 \%$ \\
Sub-total & $\mathbf{2 1}$ & $\mathbf{1 6 \%}$ \\
& Pluri-regional \\
PAL & 3 & $2.31 \%$ \\
AUST & 2 & $1.54 \%$ \\
COSM & 5 & $3.85 \%$ \\
NEO & 1 & $1.54 \%$ \\
PAN & $\mathbf{1 3}$ & $0.77 \%$ \\
ME+PAL & $130.77 \%$ \\
Sub-Total & $\mathbf{1 1 \%}$ \\
Total & $\mathbf{1 0 0 \%}$ \\
\hline \hline
\end{tabular}

then Pluriregional elements (21 species $=16 \%)$ and world-wide elements $(14$ species $=11 \%)$. In addition, the chorological affinity was chiefly made up of 78 Saharo-Sindian species, 64 species for Mediterranean taxa, 42 Irano-Turanian taxa, 14 Euro-Siberian taxa, 12 Sudano-Zambezian taxa, 5 Cosmopolitans, 4 Palaeotropical taxa, 2 species for each Neotropical and Australian taxa, and one species for Pantropical.

\section{Analysis of vegetation}

\section{Classification of stands}

Based on the importance value (out of 200) of the selected Zygophyllaceae species and their associates, classification of the 38 plotted stands using TWINSAPN led to the recognition of four community types (A, B, C and D) at the third hierarchical level (Fig. 5 ). These community types (or vegetation groups) were

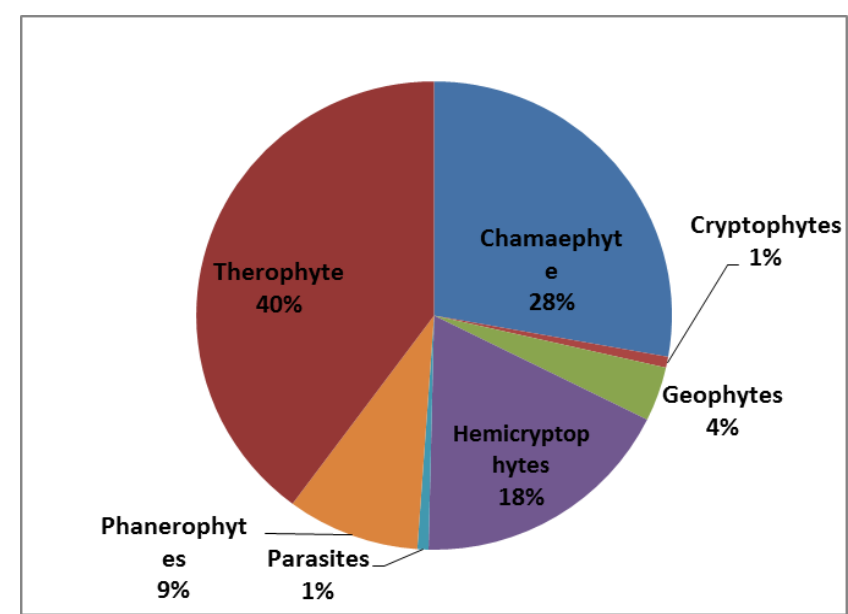

Figure (3): Life-form (\%) of the surveyed plant species in the present study.

named after the first dominant species with the highest importance value as follows: group A: Zygophyllum aegyptium, group B: Fagonia cretica, group C: Zygophyllum coccineum and group D: Pulicaria undulata.

Vegetation group A included 41 species distributed in 8 stands. It characterized sand formation habitats along Deltaic Mediterranean coastal desert. The indicator species of this group was Bromus rubens. The first and the second dominant species were Zygophyllum aegyptium and Rumex pictus. The most important associates included Senecio glaucus, Arthrocnemum macrostachyum, Reichardia tingitana and Tamarix nilotica. Group B dominated roadside habitats of the western Mediterranean coastal desert. It comprised 7 stands with 61 species. The indicator species in this group were Diplotaxis harra and Centaurea alexandrina, whereas Fagonia cretica and Avena fatua were the most dominant species. Reichardia tingitana, Mesembryanthemum crystallinum, Carthamus tenuis and Echinops spinosus represented the most important associates in this community type. Group C included 56 species in 19 stands representing Wadi Hagul bed and gravel desert habitats of Suez. It was codominated by Zygophyllum coccineum and Zilla spinosa. Ochradenus baccatus, Zygophyllum decumbens, Zygophyllum simplex and Diplotaxis harra were the most important associates, while Urospermum picroides was the indicator species in this vegetation group. At last, vegetation group D contained 18 species in 4 stands. This group inhabited wadi bed and roadside habitats in Ismailia. It was codominated by Pulicaria undulata and Zygophyllum aegyptium. The most important associated species in this community type included Haloxylon salicornicum, Fagonia arabica and Heliotropium digynum. Cleome amblyocarpa was the indicator species in this group (Table 3 ).

\section{Ordination of stands}

As shown in Fig. (6), the DCA ordination of the sampled stands was plotted on the plane of the first and second DCA axes. The vegetation groups yielded by TWINSPAN classification were clearly noticeable and distinguishable. The plot revealed a clear pattern of 


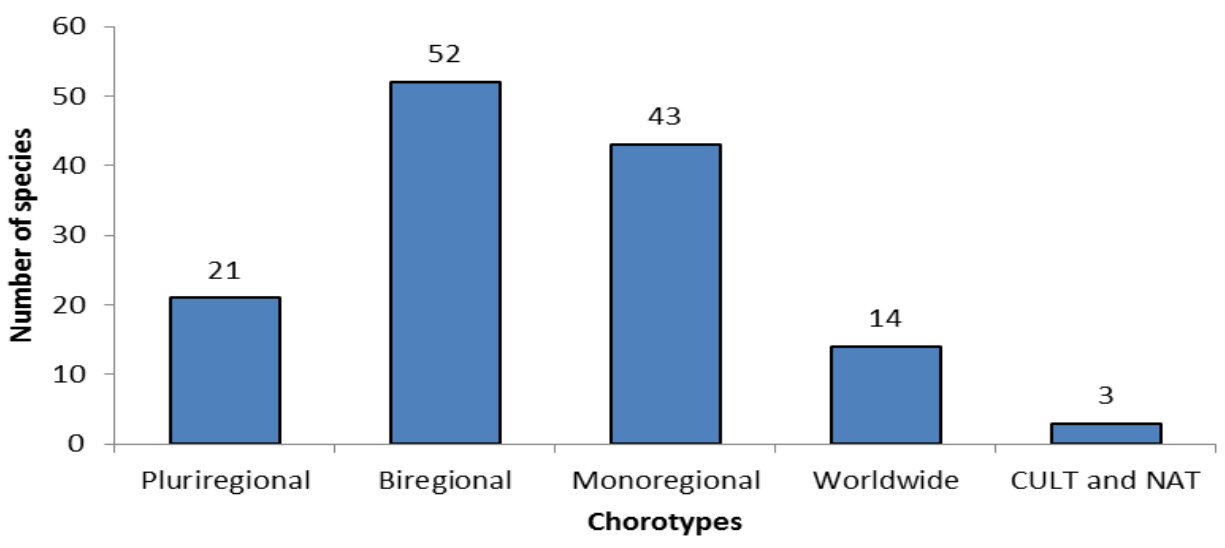

Figure (4): Chorological analysis of the surveyed plant species in the present study.

segregation of the obtained groups on the ordination plane. Groups A and D were separated at the bottom left part of DCA-diagram, while group B was segregated at the top left side. On the other hand, group $\mathrm{C}$ was detached in the right border of DCA diagram and intersected by DCA axis 2 .

\section{Vegetation-soil relationships}

Canonical Correspondence Analysis (CCA) ordination diagram was designated to illustrate the relationship between the identified vegetation groups and soil variables (Fig. 7). Eleven significant soil factors (CaCO3, organic carbon, $\mathrm{pH}$, electrical conductivity, $\mathrm{Cl}$, total nitrogen, total phosphorus, $\mathrm{Na}, \mathrm{K}, \mathrm{Ca}$ and $\mathrm{Mg}$ ) were utilized on the produced CCA-biplot. Calcium carbonate, calcium, chlorides, potassium, sodium, magnesium and EC showed the highest impact among the soil factors. These highly effective factors were correlated positively with axis 1 and axis 2 , while $\mathrm{pH}$, total nitrogen, total phosphorus and organic carbon exhibited negative correlations with both axes.

The dominant species of vegetation group $\mathrm{C}$ (Zygophyllum coccinium and Zilla spinosa) were segregated at the upper-right quarter of CCA and closely related with magnesium, potassium, chlorides, electrical conductivity and calcium carbonates. Zygophyllum aegyptium and Rumex pictus as the first and second dominant species in group $\mathrm{A}$ and the first dominant species in group D Pulicaria undulata were segregated in the lower-left quarter of CCA, and obviously correlated with organic carbon, total nitrogen, total phosphorus and $\mathrm{pH}$. On the other hand, the characteristic leading species of vegetation group B namely Fagonia cretica and Avena fatua were isolated at the upper-left quarter of CCA-diagram.

Table (3): Floristic composition features of the four vegetation groups using TWINSPAN.

\begin{tabular}{|c|c|c|c|c|c|}
\hline Group & $\begin{array}{l}\text { No. of } \\
\text { stands }\end{array}$ & $\begin{array}{l}\text { No. of } \\
\text { species }\end{array}$ & $\begin{array}{l}1^{\text {st }} \text { and } 2^{\text {nd }} \text { dominant } \\
\text { species (IV out of } \\
200)\end{array}$ & $\begin{array}{l}\text { Other important species (IV out of } \\
200 \text { ) }\end{array}$ & $\begin{array}{l}\text { Indicator } \\
\text { species }\end{array}$ \\
\hline A & 8 & 41 & $\begin{array}{l}\text { Zygophyllum } \\
\text { aegyptium }(\mathrm{IV}=49.75) \\
\text { and Rumex pictus } \\
(\mathrm{IV}=19.24)\end{array}$ & $\begin{array}{l}\text { Senecio glaucus }(\mathrm{IV}=13.88), \\
\text { Arthrocnemum macrostachyum } \\
(\mathrm{IV}=10.41), \text { Reichardia tingitana } \\
(\mathrm{IV}=8.12), \text { and Tamarix nilotica } \\
(\mathrm{IV}=8.01)\end{array}$ & $\begin{array}{l}\text { Bromus } \\
\text { rubens }\end{array}$ \\
\hline B & 7 & 61 & $\begin{array}{l}\text { Fagonia cretica } \\
(\mathrm{IV}=22.93) \text { and Avena } \\
\text { fatua }(\mathrm{IV}=15.8)\end{array}$ & $\begin{array}{l}\text { Reichardia tingitana }(\mathrm{IV}=11.93), \\
\text { Mesembryanthemum crystallinum } \\
(\mathrm{IV}=10.92), \text { Carthamus tenuis } \\
(\mathrm{IV}=9.09) \text { and Echinops spinosus } \\
(\mathrm{IV}=8.82)\end{array}$ & $\begin{array}{l}\text { Diplotaxis } \\
\text { harra and } \\
\text { Centaurea } \\
\text { alexandrina }\end{array}$ \\
\hline $\mathrm{C}$ & 19 & 56 & $\begin{array}{l}\text { Zygophyllum } \\
\text { coccineum }(\mathrm{IV}=25.58) \\
\text { and Zilla spinosa } \\
(\mathrm{IV}=24.02)\end{array}$ & $\begin{array}{l}\text { Ochradenus baccatus }(\mathrm{IV}=22.81) \\
\text { Zygophyllum decumbens }(\mathrm{IV}=18.11), \\
\text { Zygophyllum simplex }(\mathrm{IV}=8.87) \text { and } \\
\text { Diplotaxis harra }(\mathrm{IV}=8.29)\end{array}$ & $\begin{array}{l}\text { Urospermum } \\
\text { picroides }\end{array}$ \\
\hline $\mathrm{D}$ & 4 & 18 & $\begin{array}{l}\text { Pulicaria undulata } \\
(\mathrm{IV}=35.24) \text { and } \\
\text { Zygophyllum } \\
\text { aegyptium }(\mathrm{IV}=34.09)\end{array}$ & $\begin{array}{l}\text { Heliotropium salicornicum } \\
(\mathrm{IV}=30.29), \text { Fagonia arabica } \\
(\mathrm{IV}=13.42) \text { and Heliotropium } \\
\text { digynum }(\mathrm{IV}=10.99)\end{array}$ & $\begin{array}{l}\text { Cleome } \\
\text { amblyocarpa }\end{array}$ \\
\hline
\end{tabular}




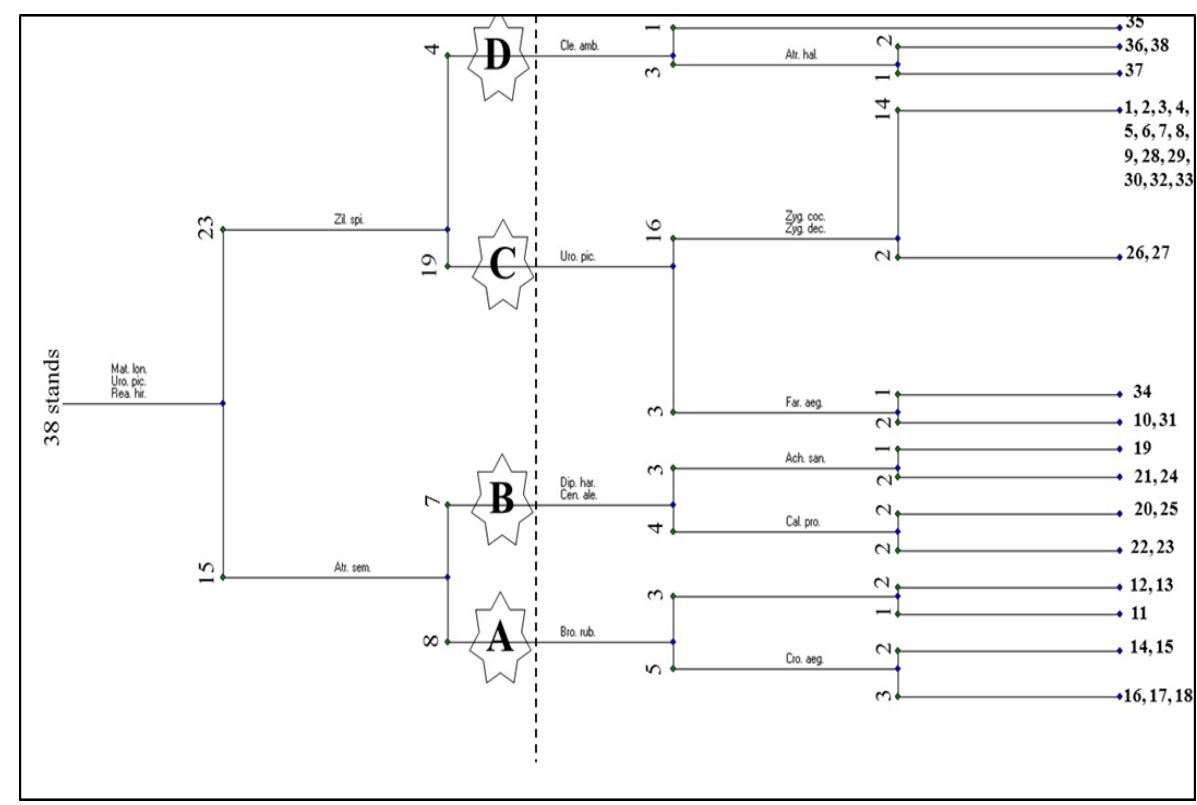

Figure. 5. TWINSPAN dendrogram displaying the four vegetation groups (A, B, C and D) at the 3rd classification level. Dashed line illustartes the level of classification. The indicator species are abbreviated by the first three letters of genus and species, respectively. The indicators species were coded as follow: Mat. lon.: Matthiola longipetala; Uro. pic.: Urpspermum picroides; Rea. hir.: Reaumuria hirtella; Zil. spi.: Zilla spinosa; Atr. sem.: Atriplex semibaccata; Cle. amb.: Cleome amblyocarpa; Dip. har.: Diplotaxis harra; Cen. ale.: Centaurea alexandrina; Bro. rub.: Bromus rubens, Atr. hal.:Atriplex halimus; Zyg. coc.: Zygophyllum coccineum; Zyg. dec.: Zygophyllum decumbens; Far. aeg.: Farsetia aegyptia; Ach. san.: Achillea santolina; Cal. pro.: Calotropis procera; and Cro. aeg.: Crotalaria aegyptiaca.

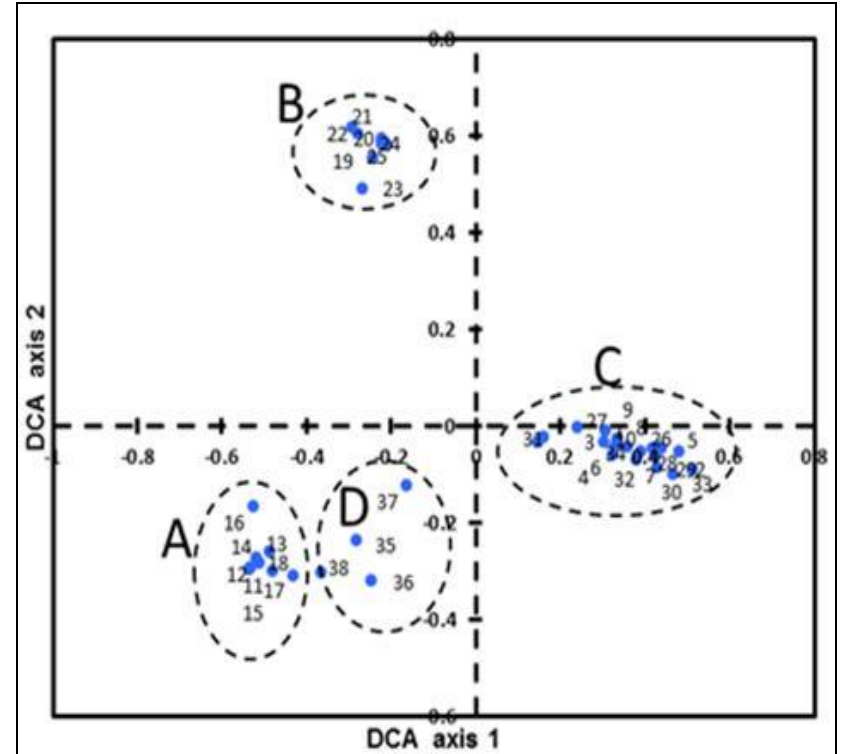

Figure 6. DCA ordination diagram of the four vegetation groups, identified by TWINSPAN classification.

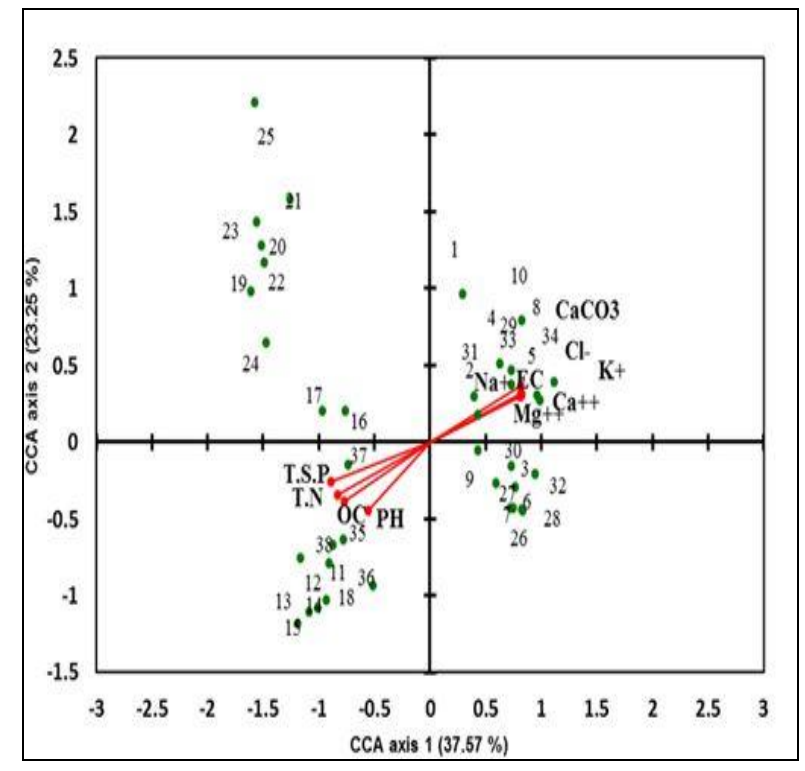

Figure. 7. CCA-ordination biplot of the sampled stands and soil variables in the present study, $\mathrm{EC}=$ Electrical conductivity, $\mathrm{OC}=$ Organic carbon, T.S.P= Total soluble phosphorus, T.N=Total nitrogen. 


\section{DISCUSSION}

The present study was aimed to investigate the spatial distribution, habitat condition, ecological aspects and chrological affinity of the selected Zygophyllaceae species with their associated plant species in some phytogeographic regions of the Egyptian desert. In Egypt, desert ecosystem is distinguished into two types of deserts; coastal and inland deserts. The coastal one is related with and adjacent to the sea coast (the Mediterranean, Red Sea or the two Gulfs of Sinai). In contrast, the inland desert incorporates the inland oases and depressions that are far from the effects of the seas (Zahran and Willis, 2009).

The floristic inventory included 133 plant species belonging to 107 genera and 29 families. About $60.15 \%$ of the investigated species were belonging to only six families namely, Asteraceae, Poaceae, Chenopodiaceae, Fabaceae, Brassicaceae and Zygophyllaceae. These results were in agreement with the studies of El-Amier et al. (2015) and Mashaly et al. (2016).

Asteraceae ranked first followed by Poaceae then Chenopodiaceae, which together account for $34 \%$ of the Egyptian flora (Boulos 2009), while Fabaceae, Brassicaceae and Zygophyllaceae were the last among the six families with 9 species for each. In the present study, the life-form spectrum in the surveyed area is predominantly therophytic species. The predominance of therophytes is an indicator of the prevailing arid climate as those elements are characterized by high reproductive capacity, ecological and genetic plasticity and high adaptation to mild moist winter and hot dry summer. This has been confirmed by Salama et al. (2013), Abd El-Ghani et al. (2014), Abd El-Aal et al. (2015) and Mashaly et al. (2016). In addition, the dominance of perennials $(60 \%$ of total surveyed taxa) may be related to the nature of the habitat types in the study area in which the reproductive capacity, morphological, ecological and genetic plasticity are the limiting factors (Grime ,2006). These results show the influence of climate topographic, hot-arid variation and biotic factors in the study area (Shaltout et al., 2015).

The current study was mainly regarded as a mixture of the chorotypes belonging to Saharo-Sindian, Mediterranean and Irano-Turanian regions. The effect of these phytogeographical zones was highly reflected in the flora of the study area. The Preponderance of Saharo-Sindian chorotypes could be attributed to the location of Egypt in the center of Saharo-Sindian region (Barakat et al., 2014). The other floristic elements such as Euro-Siberian, Sudano-Zambezian, Cosmopolitans, Palaeotropical, Neotropical, Australian and Pantropical were in a varying miniature representation reflecting their differential capability to penetrate the region (Shaltout et al., 2015).

The yielded four vegetation groups using TWINSPAN classification and DCA ordination reflect the different habitats in the study area. These groups were named after the first dominant species. Group A: Zygophyllum aegyptium, group B: Fagonia cretica, group C: Zygophyllum coccineum and group D: Pulic- aria undulate..

Groups A and B represented the surveyed coastal desert, while $\mathrm{C}$ and D communities reflect the inland Eastern desert. Zygophyllum aegyptium community may stand for the sand formation habitats along Deltaic Mediterranean coastal desert. In addition, Fagonia cretica community dominated the roadside habitats of the western Mediterranean coastal desert. On the other hand, Zygophyllum coccineum community represented Wadi Hagul bed and gravel desert habitats of Suez. At last, Pulicaria undulata community inhabited wadi bed and roadside habitats in Ismailia. This agrees more or less, with the findings of Abd El-Ghani et al. (2014), Mashaly et al. (2015 \& 2016).

Results of CCA-biplot of species and soil factors indicated that soil factors especially calcium carbonate, calcium, chlorides, potassium, sodium, magnesium and EC showed the highest impact among the soil factors that affecting the distribution and abundance of the surveyed plant species. The dominant species of vegetation group $\mathrm{C}$ (Zygophyllum coccinium and Zilla spinosa) were segregated at the upper-right quarter of CCA and closely related with magnesium, potassium, chlorides, electrical conductivity and calcium carbonates. Zygophyllum aegyptium and Rumex pictus as the first and second dominant species in group A and the first dominant species in group D Pulicaria undulata were segregated in the lower-left quarter of CCA, and obviously correlated with organic carbon, total nitrogen, total phosphorus and $\mathrm{pH}$. On the other hand, the characteristic leading species of vegetation group $\mathrm{B}$ namely Fagonia cretica and Avena fatua were isolated at the upper-left quarter of CCA-diagram. These results were in agreement with the findings of Alattar (2018) and Showran (2018).

\section{Acknowledgement}

The authors would like to thank the Academy of Scientific Research and Technology, Ministry of Scientific Research for supporting Mona Elmorsy by the grant of Scientists for Next Generation (ASRT/SNG/FA/2014-9).

\section{REFERENCE}

ABD ELAAL, M., H. M. EL-SHORA AND O. J. KADHIM. 2015. Community Associates of the Medicinal Farsetia Aegyptia Turra and its Soil Correlates in the North-Eastern Desert of Egypt. Mansoura Journal of Biology, 40:130-142.

ABD EL-GHANI, M. M., HUERTA-MARTÍNEZ, F. M., L. Hongyan and R.Qureshi. 2017. Plant Responses to Hyperarid Desert Environments. Springer International Publishing.

ABD EL-GHANI, M. M., SALAMA, F., SALEM, B., EL HADIDY, A. AND ABDEL-ALEEM, M. 2014. Biogeographical relations of a Hyper Arid Desert Flora in Eastern Egypt. African Journal of Ecology, 52:173-191

ABDEL KHALIK, K. N. 2012. A Numerical Taxonomic Study of the Family Zygophyllaceae from Egypt. Acta Botanica Brasilica, 26: 165-180. 
ABDELAAL, M. 2017. Current Status of the Floristic Composition in Wadi Hagul, Northwest Suez Gulf, Egypt. Rendiconti Lincei, 28(1): 81-92.

ALATTAR, A. Y. 2018. Ecological Features and Economic Potentialities of Some Species of Family Boraginaceae in the Mediterranean Coast, Egypt. M. Sc. Thesis, Faculty of Science, Mansoura University, Egypt.

AOAC. 1990. Official Methods of Analysis, 15th Edition. Association of Official Analytical Chemists, Arlington, Virginia.

BARAKAT, N. A. M., A. M. A. EL-GAWAD, V. LAUDADIO, H. F. KABIEL, V. TUFARELLI, AND E.CAZZATO. 2014. A Contribution to the Ecology and Floristic Markers of Plant Associations in Different Habitats of Sinai Peninsula, Egypt. Rendiconti Lincei, 25(4): 479-490.

BEIER, B. A., J. A. A. NYLANDER, M. W. CHASE AND M.THULIN. 2004. Phylogenetic relationships and biogeography of the Desert Plant Genus Fagonia (Zygophyllaceae), inferred by Parsimony and Bayesian Model Averaging. Molecular Phylogenetics and Evolution, 33(1): 91-108.

BEIER, B. A., M. W. CHASE AND M. THULIN. 2003. Phylogenetic Relationships and Taxonomy of Subfamily Zygophylloideae (Zygophyllaceae) Based on Molecular and Morphological Data. Plant Systematics and Evolution, 240(1-4): 11-39.

BOULOS L. 1999. Flora of Egypt. AzollaceaeOxalidaceae, Vol 1. Al Hadara, Cairo, p 419.

BOULOS L. 2000. Flora of Egypt. GeraniaceaeBoraginaceae, Vol 2. Al Hadara, Cairo, p 352.

BOULOS L. 2002. Flora of Egypt. VerbinaceaeCompositae, Vol 3. Al Hadara, Cairo, p 373.

BOULOS L. 2005. Flora of Egypt. Monocotyledons (Alismataceae- Orchidaceae), Vol 4. Al Hadara, Cairo, p 617.

BOULOS L. 2009. Flora of Egypt Checklist, Revised Annotated Edition. Al Hadara, Cairo.

EL GHOUL, J., M. SMIRI, S. GHRAB, N. A BOUGHATTAS. AND M. BEN-ATTIA, 2012. Antihyperglycemic, Antihyperlipidemic and Antioxidant activities of Traditional Aqueous Extract of Zygophyllum album in Streptozotocin Diabetic Mice. Pathophysiology, 19(1): 35-42.

EL HADIDI, M. N. 1966. The Genus Fagonia L. Egypt. Candollea, 21(1): 13-54.

EL HADIDI, M. N. 1972. The family Zygophyllaceae in Egypt: 1. Fagonia L. and Seetzenia R. Br. Botaniska Notiser, 125(4): 523-535.

EL-AMIER, Y. A., E. S. F. EL-HALAWANY, S. A. HAROUN, AND S. G. MOHAMUD. 2015. Vegetation Analysis and Soil Characteristics on Two Species of Genus Achillea Growing in Egyptian Desert. Open Journal of Ecology, 5: 420-433.

ELBADRY, M. A., M. M. ELAASSER, H. H. ELSHIEKH, AND M. M. SHERIFF. 2015. Evaluation of Antimicrobial, Cytotoxic and larvicidal Activity of Zygophyllum coccineum North Sinai, Egypt. Med Aromat Plants, 4(214): 2167 0412 .
ENGLER A. 1896. Uber Die Geographische Verbreitung Der Zygophyllaceen Im Verhaltniss $\mathrm{Zu}$ Ihrer Systematischen Gliederung. Abhandlungen Der Preussischen Akademie Der Wissenschaften, Physikalisch-Mathematische Klasse, Berlin.

ENGLER A. 1931. Zygophyllaceae, Rutaceae, Simaroubaceae, Burseraceae. in: Engler A, Prantl K (eds) Die natürlichen Pflanzenfamilien, 2nd edn, Vol 19a. Engelmann, Leipzig, pp 144-184, 187-456.

FARHEEN, R., MAHMOOD, I., PARVEEN, R., KISHWAR, F. AND ANWAR, A. 2017. Review on Medicinal and Bioactive Role Of Genus Fagonia. FUUAST Journal Of Biology, 7(1): 33-36. FEINBRUN-DOTHAN, N. 1978. Flora Palaestina: Part 3. Ericaceae to Compositae. Jerusalem, Israel Academy of Sciences and Humanities. 481.

FEINBRUN-DOTHAN, N.1986. Flora Palestina, Part 4. Israel Acad Sciences and Humanities, Jerusalem. Israel Academy of Sciences and Humanities. 463.

GIBBONS, S. AND M. A. ORIOWO. 2001. Antihypertensive Effect of an Aqueous Extract of Zygophyllum coccineum L. in Rats. Phytotherapy Research, 15(5): 452-455.

GRIME, J. P. 2006. Plant Strategies, Vegetation Processes, and Ecosystem Properties. John Wiley \& Sons.

HENDERSON P. A. AND R. M. SEABY 2007. Community Analysis Package 4.0. Pisces Conservation Ltd, Lymington.

LAM, M., A. R. CARMICHAEL AND H. R. GRIFFITHS. 2012. An Aqueous Extract of Fagonia cretica Iinduces DNA Damage, Cell Cycle Arrest and Apoptosis in Breast Cancer Cells Via FOXO3a and p53 Expression. PLoS ONE, 7(6): e40152.

MASHALY, I. A. 2001. Contribution to the Ecology of the Deltaic Mediterranean Coast, Egypt. Online Journal of Biological Sciences, 1 (7): 628-635.

MASHALY, I. A. 2002. Ecological Studies on Zygophyllum aegyptium in the Deltaic Mediterranean Coast of Egypt. Pakistan Journal of Biological Sciences, 5 (2): 152-160.

MASHALY, I. A., M. ABD EL-AAL, H.S. ALDESUQUY AND B.A. MAHDEE. 2016. Floristic Perspective for Some Medicinal Plants Growing in the Coastal and Inland Deserts of Egypt. International Journal of Current Research, 8: $24917-$ 24925.

MASHALY, I. A., Y. EL-AMIER AND A.YAHIA. 2015. Phytosociological and ecological studies of five species of genus Zygophyllum in Egypt. Mansoura Journal of Biology, 40: 79-96.

MUELLER-DOMBOIS, D. AND H. ELLENBERG. 1974. Aims and Methods of Vegetation Ecology.John Willey and Sons, New York, Chichester Brisbone, Toranto.

PAREEK, A., A. GODAVARTHI, R. ISSARANI AND B. P. NAGORI. 2013. Antioxidant and Hepatoprotective Activity of Fagonia schweinfurthii (Hadidi) Hadidi Extract in Carbon Tetrachloride induced Hepatotoxicity in HepG2 Cell 
line Andrats. Journal of Ethnopharmacology, 150(3): 973-981.

QURESHI, H., S. ASIF, H. AHMED, H. A. ALKAHTANI AND K. HAYAT. 2016. Chemical Composition and Medicinal Significance of Fagonia cretica: A Review. Natural Product Research, 30(6): 625-639.

RAUNKIAER, C. 1934. The Life Forms of Plants and Statistical Plant Geography. The Clarendon Press.

SALAMA, F. M., M. M. A.. EL-GHANI, S. M. ELNAGGAR,. AND M. M. ALJARROUSHI. 2013. Vegetation Analysis and Species Diversity in the Desert Ecosystem of Coastal Wadis of South Sinai, Egypt. Journal of Biology and Earth Sciences, 3(2): 214-227.

SHALTOUT, K. H., H. A. HOSNI, R. A.EL-FAHAR, AND D. A. AHMED. 2015. Flora and Vegetation of the Different Habitats of the Western Mediterranean Region of Egypt. Taeckholmia, 35: 45-76.

SHEAHAN, M. C. AND D. F.CUTLER. 1993. Contribution of Vegetative Anatomy to the Systematics of the Zygophyllaceae R. Br. Botanical Journal of the Linnean Society, 113(3): 227-262.

SHEAHAN, M. C. AND M. W CHASE, 2000. Phylogenetic Relationships Within Zygophyllaceae Based on DNA Sequences of Three Plastid Regions, with Special Emphasis on Zygophylloideae. Systematic Botany, 25(2):371-384.

SHEAHAN, M. C. AND M. W. CHASE. 1996. A Phylogenetic Analysis of Zygophyllaceae R. Br.
Based on Morphological, Anatomical and rbc L DNA Sequence Data. Botanical Journal of the Linnean Society, 122(4): 279-300.

SHOWRAN, R. A. 2018. Ecological Study on the Desert Shrub Ochradenus baccatus Delile in Wadi Hagul, North Eastern Desert, Egypt. M. Sc. Thesis, Faculty of Science, Mansoura University, Egypt.

TACKHOLM, V. 1974. Students' Flora of Egypt. Beirut, Cairo University.

TER BRAAK, C. F. 2003. CANOCO, Version 4.52. Wageningen University and Research Centre, Wageningen.

UPADHYAY, R. M. AND N. L. SHARMA.2005. Manual of Soil, Plant, Water and Fertilizer Analysis. Kalyani, New Delhi.

WALKLEY, A., AND I. A. BLACK. 1934. An Examination of the Degtjareff method for

Determining Soil organic Matter, and A Proposed Modification of the Chromic Acid Titration Method. Soil Sci., 37:29-38.

WILDE, S. A., G. K. VOIGT AND J. G.LYER. 1964. Soil and Plant Analysis for Tree Culture. Edited by Gordon Chesters, Applied Soil Science Publisher.

ZAHRAN, M.A. AND A.J. WILLIS. 2009. The Vegetation of Egypt.2nd ed. Springer. Netherlands.

ZOHARY, M. 1966. Flora Palaestina, Part 1. The Israel Academy of Sciences and Humanities, Jerusalem, 364.

ZOHARY, M. 1972. Flora Palaestina, Part 2. The Israel Academy of Sciences and Humanities. Jerusalem, 203.

\title{
بيئة الغطاء الخضري للنباتات المرافقة لبعض أنواع الفصيلة الرطريطية في مناطق جغرافية مختلفة من

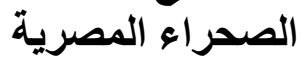
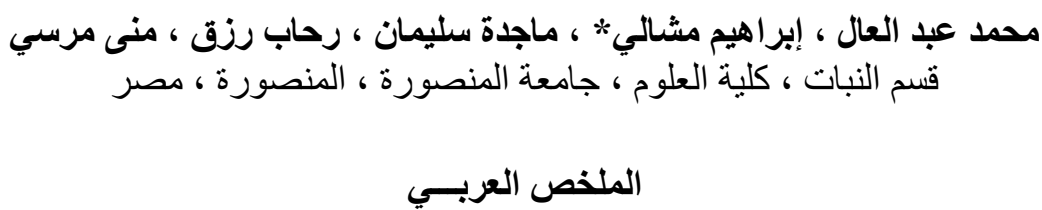

\begin{abstract}
تعد العائلة الرطر اطية فصيلة نباتية واسعة الانتشار تتميز بوجود شائع فى البيئات الجافة والثبه جافة في كل من العالمين القديم والجديد وبشكل خاص فى الصحاري الجافة موسميا.وقد اختيرت فى هذه الدراسة ثمانية أنواع من العائلة الرطراطية وهي:الثويكة Fagonia Fagonia bruguieriv، عاقول الغزال Fagonia cretica mollis،arabica Zygophyllum aegyptium البطباط Zygophyllum coccineum ، الرطريط المفلطح Zygophyllum decumbens، القرمل تمت در اسة السمات الفلورية للانو اع النباتية المختارة و المجتمعات النباتية المصاحبة لها ودر اسة مدى تأثر ها بعوامل التربة المختلفةحيث تم اختيار 38 موقعا فى صحر اء مصر الثرقية والصحر اء الساحلية المطلة على ساحل البحر المتوسط. أظهرت نتائج الدراسة تسجيل 133 نوعا من النباتات تتبع 107 جنسا وتنتمي إلى 29 عائلة زهرية ووجد أن الفصائل: المركبة والنجيلية والرمر امية والصليبية والقرنية و الرطريطية

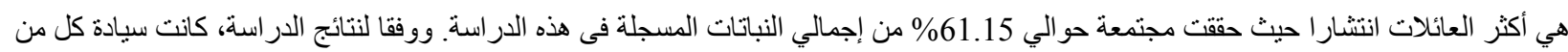
النباتات المعدرة (60\%)و النباتات من طراز الحوليات (40\%) وكذلك سيادة النباتات التى تتبع إقليم الصحاري ـالسندي فى الجنوب والتي تتبع إقليم البحر المتوسط معا(62\%) دليلا معبرا على المناخ الجاف والثبه جاف لمنطقة الدراسة. ولتقدير وفرة الأنواع النباتية بالعشائر المختلفة فى منطقة الدراسة تم تطبيق برامج التصنيف والتسلسل ثنائي الاتجاه (TWINSPAN) على البيانات الخاصة بالوفرة والتي تتمثل فى قيمة الأهية
\end{abstract}


(A, B, C and D) وذلك حتى يمكن التعرف على المجمو عات النباتية وقد أمكن التعرف على أربعة مجمو عات من النباتات(Importance value)

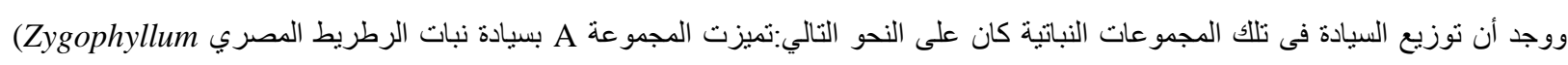

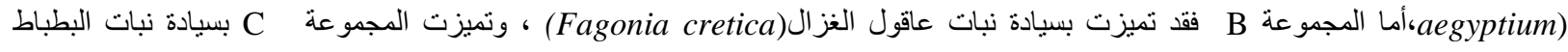

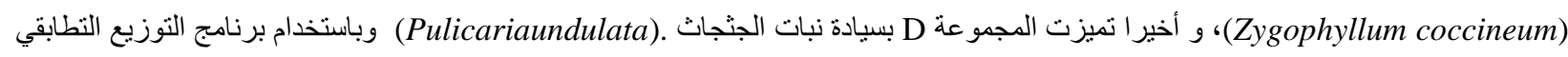
العكسي (DCA) أمكن فصل المجموعات النباتية الناتجة باستخدام برنامج التصنيف و التسلسل ثنائي الاتجاه. وباستخدام برنامج تحليل التطابق

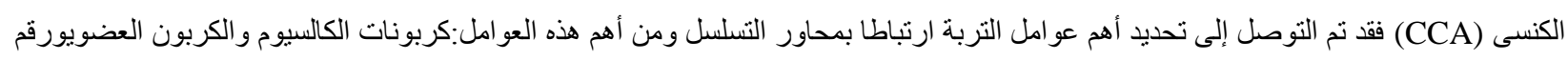
الأس الهيدروجيني و التوصيل الكهربائي والكلوريدات و النيتروجين الكلي والفسفور الكلي الذائب والعناصر المعدنية الصوديوم، البوتاسيوم، الكالسيوم، 